\title{
Summary of the CKM 2016 working group on rare decays
}

\author{
Akimasa Ishikawa \\ Department of Physics, Tohoku University, Sendai, Japan \\ E-mail: akimasa@epx.phys.tohoku.ac.jp
}

\section{Enrico Lunghi}

Physics Department, Indiana University, Bloomington IN, USA

E-mail: elunghi@indiana.edu

\section{Matthew Moulson}

INFN Laboratori Nazionali di Frascati, Italy

E-mail: Matthew.Moulson@lnf.infn.it

\section{Justine Serrano}

Aix Marseille Univ, CNRS/IN2P3, CPPM, Marseille, France

E-mail: serrano@cppm.in2p3.fr

Rare $B, D$, and $K$ decays offer unique opportunities to probe for evidence of new particles from physics beyond the Standard Model at mass scales extending from the electroweak scale to well above those directly accessible at the LHC. We review a selection of theoretical and experimental results on these decays illustrating the progress made during the past two years.

9th International Workshop on the CKM Unitarity Triangle

28 November - 3 December 2016

Tata Institute for Fundamental Research (TIFR), Mumbai, India 


\section{Introduction}

The presence of a single Higgs doublet in the Standard Model of particle physics (SM) implies that all flavour-changing transitions are determined by the three angles and the one phase that parameterize the Cabibbo-Kobayashi-Maskawa matrix (CKM) [1,2]. Rare $B, D$, and $K$ decays proceed at loop level via vertices suppressed by small, off-diagonal CKM entries, thus offering exquisite tests of the Higgs mechanism of electroweak symmetry breaking.

Deviations from SM predictions are usually parameterized in terms of non-standard contributions to various non-renormalizable operators whose intrinsic scale cannot be too far removed from the electroweak scale in order to produce observable effects. While this usually means that non-standard contributions to rare decays are associated with new particles that are within the reach of the LHC, there are some observables (especially involving kaons) that are sensitive to energy scales that are far beyond current collider capabilities.

During the past two years there has been enormous experimental progress, with ground breaking analyses from LHCb, ATLAS, CMS, BaBar, and Belle. The general picture that emerges is an overall agreement with SM expectations. However, there are several observables for which measurements are in some tension with the SM. Confirmation of these discrepancies would point to the existence of new massive particles that are possibly within the reach of ATLAS and CMS and which would very likely introduce novel non-CKM like flavour changing interactions. The most notable anomalies appear in $B \rightarrow K^{(*)} \ell \ell$ and $B \rightarrow D^{(*)} \ell v$ decays.

The upcoming high luminosity $B$-factory Belle II is about to come online, promising important cross checks of these effects [3]. Moreover, the NA62 [4] and KOTO [5, 6] experiments are also taking data and will offer precise measurements of the extremely rare kaon decays $K^{+} \rightarrow \pi^{+} v \bar{v}$ and $K_{L} \rightarrow \pi^{0} v \bar{v}$, whose branching ratios can be calculated with an incredible theoretical accuracy.

In the following, we review some of the recent theoretical and experimental progresses that have been made during the last two years in $\operatorname{rare} B, D$, and $K$ decays.

\section{Rare $b$-hadron decays}

Rare flavor-changing neutral current (FCNC) decays of $b$-hadrons can be described in a modelindependent approach using the effective Hamiltonian

$$
\mathscr{H}_{\mathrm{eff}}=-\frac{4 G_{F}}{\sqrt{2}} V_{t b} V_{t q}^{*} \sum_{i}\left(C_{i} \mathscr{O}_{i}+C_{i}^{\prime} \mathscr{O}_{i}^{\prime}\right)+\text { h.c. }
$$

where $G_{F}$ is the Fermi coupling constant and $V_{t b, t q}$ are the CKM matrix elements with $q=d, s$ for processes based on the quark level $b \rightarrow d, s$ transitions. The heavy degrees of freedom have been integrated out in the short distance Wilson coefficients $C_{i}$, and the operators $\mathscr{O}_{i}$ encode the long-distance effects. In the SM, the main operators are the electromagnetic operator $\mathscr{O}_{7}$ and the semileptonic operators $\mathscr{O}_{9}$ and $\mathscr{O}_{10}$. New-physics (NP) contributions could affect the value of the Wilson coefficients $C_{7,9,10}$ or involve other operators such as $\mathscr{O}_{7,9,10}^{\prime}$ or $\mathscr{O}_{S, P}^{(\prime)}$. The different $\mathscr{O}_{i}$ contribute differently to leptonic, semileptonic, and radiative decays. The next three sections present the experimental results regarding these channels, while Section 2.4 presents a global analysis of these measurements in the effective Hamiltonian framework. 


\subsection{Leptonic decays}

The $B_{d}^{0} \rightarrow \mu^{+} \mu^{-}$and $B_{s}^{0} \rightarrow \mu^{+} \mu^{-}$channels are particularly sensitive to NP contributions in the scalar/pseudoscalar sector and have been searched for for more than 25 years. The results of a combined analysis of the CMS and LHCb Run 1 data were presented at CKM 2014, revealing the first observation of the $B_{s}^{0} \rightarrow \mu^{+} \mu^{-}$decays. The measured branching ratios are compatible with SM expectations, $\operatorname{BR}\left(B_{s}^{0} \rightarrow \mu^{+} \mu^{-}\right)=(3.65 \pm 0.23) \times 10^{-9}$ and $\operatorname{BR}\left(B_{d}^{0} \rightarrow \mu^{+} \mu^{-}\right)=(1.06 \pm 0.09) \times$ $10^{-10}$ [7], at the $1.2 \sigma$ level for the $B_{s}^{0}$ and $2.2 \sigma$ for the $B_{d}^{0}$ decays. The ATLAS collaboration recently presented the results of its analysis of Run 1 data, $\operatorname{BR}\left(B_{s}^{0} \rightarrow \mu^{+} \mu^{-}\right)=\left(0.9_{-0.8}^{+1.1}\right) \times 10^{-9}$ and $\operatorname{BR}\left(B^{0} \rightarrow \mu^{+} \mu^{-}\right)<4.2 \times 10^{-10}$ at $95 \%$ confidence level (CL) [8], which are in agreement with the $\mathrm{CMS}+\mathrm{LHCb}$ combination. The analysis of the data taken by the LHC experiments during Run 2 will improve these measurements and start to provide additional observables such as the ratio of the $B_{d}^{0}$ and $B_{s}^{0}$ modes, which is sensitive to Minimal Flavour Violation scenarios, or the $B_{s}^{0} \rightarrow \mu^{+} \mu^{-}$effective lifetime, which has different sensitivity to NP models with scalar and nonscalar contributions.

Searches for leptonic $B$ decays into $\tau$ leptons are interesting in view of the recent hints of lepton flavour non-universality obtained by several experiments. Their branching ratios are two orders of magnitude higher than those for decays into muons because of the less stringent helicity suppression and the higher lepton mass: $\operatorname{BR}\left(B_{s}^{0} \rightarrow \tau^{+} \tau^{-}\right)=(7.73 \pm 0.49) \times 10^{-7}$ and $\operatorname{BR}\left(B_{d}^{0} \rightarrow \tau^{+} \tau^{-}\right)=$ $(2.22 \pm 0.19) \times 10^{-8}$ [7]. However, experimental searches for these decays are complicated by the presence of at least two neutrinos in the final state from the $\tau$ decays. The LHCb Collaboration has presented preliminary results corresponding to the first limit on $\operatorname{BR}\left(B_{s}^{0} \rightarrow \tau^{+} \tau^{-}\right)$, at $3.0 \times 10^{-3}$ (95\% CL), and the best limit on $\operatorname{BR}\left(B_{d}^{0} \rightarrow \tau^{+} \tau^{-}\right.$), at $1.3 \times 10^{-3}$ (95\% CL) [9], obtained using the hadronic tau decay $\tau^{+} \rightarrow \pi^{-} \pi^{+} \pi^{-} v_{\tau}$.

\subsection{Semileptonic decays}

Rare semileptonic decays provide two classes of observables allowing tests of the SM. The observables in the first one provide tests of lepton flavour universality and are theoretically very clean. An example is the measurement of the ratio $R_{K}=\Gamma\left(B^{+} \rightarrow K^{+} \mu^{+} \mu^{-}\right) / \Gamma\left(B^{+} \rightarrow K^{+} e^{+} e^{-}\right)$, which was found to be $R_{K}=0.745_{-0.074}^{+0.090} \pm 0.036$ by LHCb in the range $1<q^{2}<6 \mathrm{GeV}^{2} / c^{4}$ [10], 2.6 $\sigma$ lower than the SM prediction, $1.00 \pm 0.01[11]\left(q^{2}\right.$ is the squared invariant mass of the dilepton system). A new BaBar analysis performed in the same $q^{2}$ region confirmed the deficit in the muonic mode, measuring $R_{K}=0.64_{-0.30}^{+0.39} \pm 0.06$.

The observables in the second class include differential branching ratios and angular distributions. Their theoretical predictions are affected by hadronic uncertainties arising from the form factors, which are computed using lattice QCD or light cone sum-rule techniques, depending on the $q^{2}$ region. An interesting picture emerges from the differential branching ratio measurements for $b \rightarrow s \mu^{+} \mu^{-}$decays from the LHCb Collaboration, in which, below the charmonium resonances, the experimental values tend to be lower than the SM predictions [12, 13, 14, 15], in agreement with the deficit in the muonic mode seen in $R_{K}$. The largest effect is seen in the $B_{s} \rightarrow \phi \mu^{+} \mu^{-}$ channel, where the discrepancy is at the level of $3 \sigma$.

The angular observables for $B \rightarrow K^{*} \ell^{+} \ell^{-}$decays, where $\ell=e, \mu$, have been studied by several 

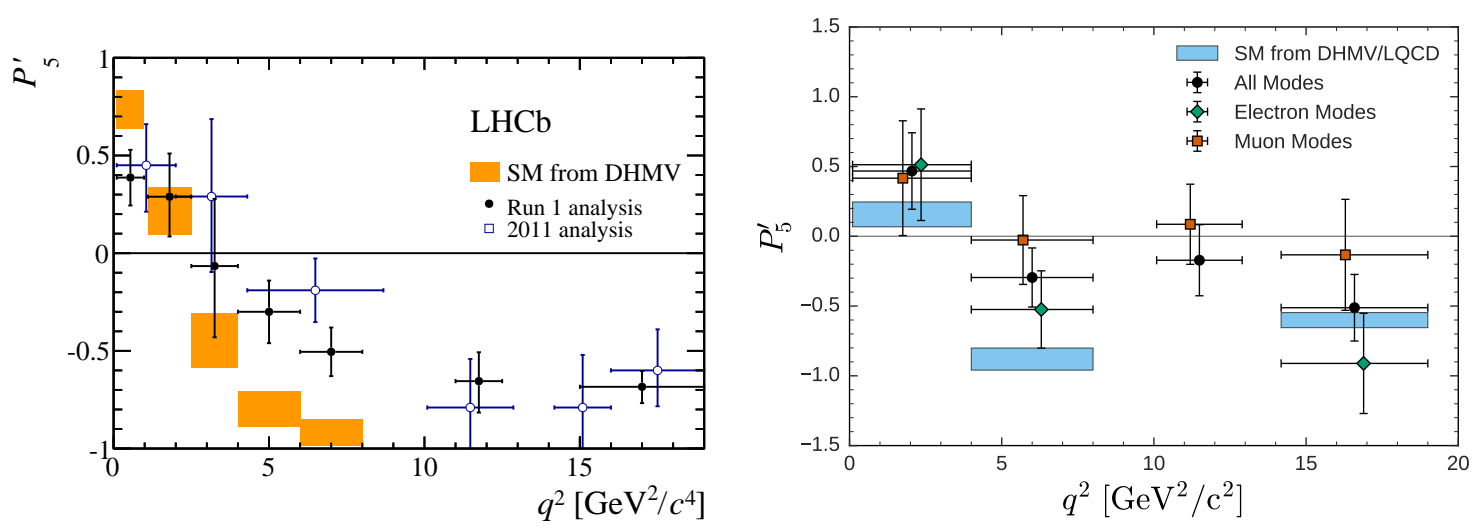

Figure 1: The $P_{5}^{\prime}$ angular observables in bins of $q^{2}$. The shaded boxes show the SM prediction taken from [16]. Left: LHCb results from $B^{0} \rightarrow K^{* 0} \mu^{+} \mu^{-}$data. Right: Belle results from $B^{(+)} \rightarrow K^{*(+)} \ell^{+} \ell^{-}$data for electron modes, muon modes, and the combination.

experiments $[17,18,19,20]$. The differential decay rate can be described by

$$
\frac{1}{d \Gamma / d q^{2}} \cdot \frac{d^{4} \Gamma\left[\bar{B}^{0}+B^{0}\right]}{d \cos \theta_{\ell} d \cos \theta_{K^{*}} d \phi d q^{2}}=\frac{9}{32 \pi} \sum_{i} S_{i}\left(q^{2}\right) f_{i}\left(\cos \theta_{\ell}, \cos \theta_{K^{*}}, \phi\right),
$$

where $\theta_{\ell}$ is the angle between the negatively charged lepton and the incoming $B$ direction in the dilepton system center-of-mass frame, $\theta_{K^{*}}$ is the angle between the Kaon and the incoming $B$ direction in the $K$ - $\pi$ system center-of-mass frame, $\phi$ is the angle between the $B \ell \ell$ and $B K \pi$ planes and the observables $S_{i}$ are functions of the Wilson coefficients. Other observables with reduced sensitivity to the hadronic uncertainties can be formed from the $S_{i}$, in particular $P_{5}^{\prime}=\frac{S_{5}}{\sqrt{F_{L}\left(1-F_{L}\right)}}$, where $F_{L}$ is the fraction of longitudinally polarized $K^{*} \mathrm{~s}$.

Using the Run 1 data set of $3 \mathrm{fb}^{-1}$, the LHCb Collaboration has performed a full angular analysis of the $B^{0}$ decay, confirming the tension previously seen with $1 \mathrm{fb}^{-1}$ between the measurements of the $P_{5}^{\prime}$ observables and SM predictions at low $q$, as shown in Fig. 1, left [19]. This tension is now at the $3.4 \sigma$ level. The Belle Collaboration has used a folding technique to access the same observable, combining the $B^{0}$ and $B^{+}$modes, and reported a tension of $2.6 \sigma$ in the bin $4<q^{2}<8 \mathrm{GeV}^{2} / c^{4}$ [20]. They also measured for the first time the $Q_{i}=P_{i}^{\mu}-P_{i}^{e}$ variables introduced in [21], which also provide a test of lepton flavour universality.

The anomalies in $R_{K}$ and $P_{5}^{\prime}$, together with the pattern of neutrino mixings, can be simultaneously explained by NP in the operators $\mathscr{O}_{9}^{\ell}$ and $\mathscr{O}_{9}^{\prime \ell}$. The NP can be introduced via the $Z^{\prime}$ of a $U(1)_{X}$ symmetry [22]. A model can be constructed that satisfies the constraints from $B$ and $K$ mixing and rare $B$ decays, as well as from direct searches for $p p \rightarrow Z^{\prime} \rightarrow \mu \mu$ at colliders. Direct detection of a $4 \mathrm{TeV} Z^{\prime}$ in the $\mu \mu$ channel at the LHC would require several hundred $\mathrm{fb}^{-1}$.

The $b \rightarrow(s, d) v \bar{v}$ transitions are theoretically cleaner than the modes with charged leptons, as only the $Z$ boson can intervene in the penguin diagram. According to the SM, the predicted branching ratios span a range from $2.4 \times 10^{-7}$ for the $\pi^{+}$mode [23] to $9.2 \times 10^{-6}$ for the $K^{*+}$ mode [24]. Searches for these decay channels have been performed by the Belle and BaBar experiments using full-event reconstruction thanks to hadronic [25, 26] or semileptonic tagging modes [27]. At this conference, the Belle Collaboration presented new results based on an improved semileptonic 
tagging method leading to the most stringent limits on $B^{0} \rightarrow K_{s}^{0} v \bar{v}, B^{0} \rightarrow K^{* 0} v \bar{v}, B^{+} \rightarrow \pi^{+} v \bar{v}$, $B^{0} \rightarrow \pi^{0} v \bar{v}, B^{+} \rightarrow \rho^{+} v \bar{v}$ and $B^{0} \rightarrow \rho^{0} v \bar{v}$ [28], as seen in Fig. 2. It should be noted that numerous

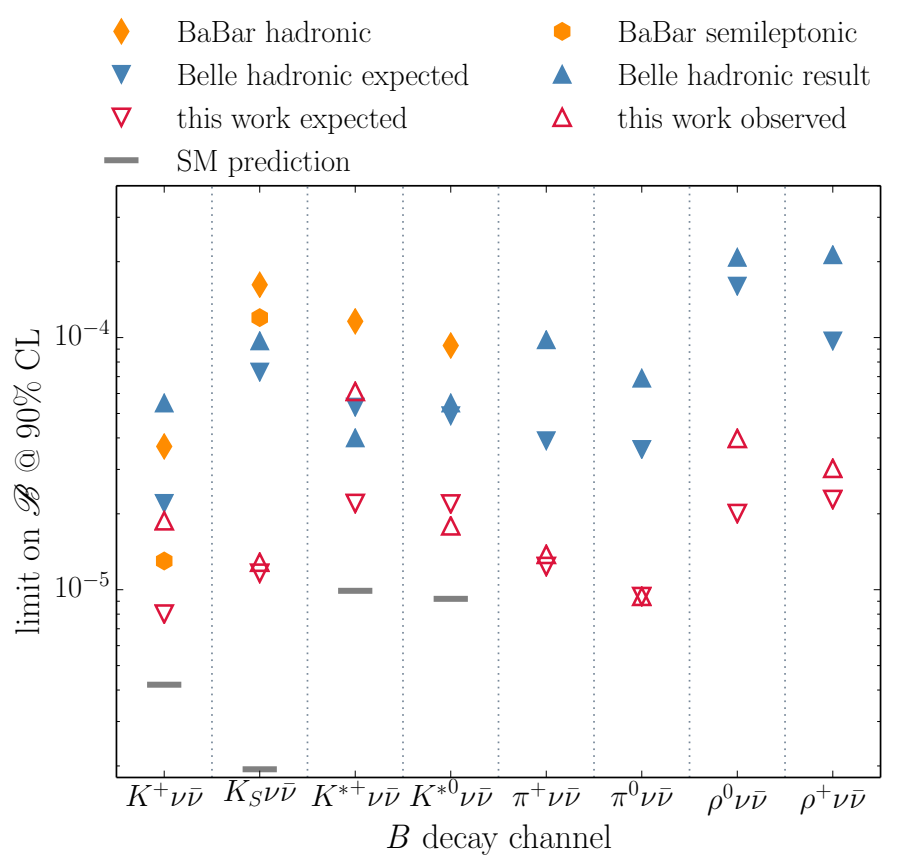

Figure 2: Expected and observed limits obtained in [28] in comparison to previous results for the BaBar measurement with semileptonic [27] and hadronic tag [26], as well as the Belle measurement utilizing hadronic tagging [25]. Theory predictions are from [24]. The processes $B \rightarrow(\pi, \rho) v \bar{v}$ are suppressed by $\left|V_{t d} / V_{t s}\right|^{2}$ with respect to $B \rightarrow K^{(*)} v \bar{v}$ and have branching ratios below $10^{-6}$ [29].

other new results on $b \rightarrow(s, d) \ell \ell$ transitions have appeared since the last CKM workshop in 2014, such as the first observation of a $b \rightarrow d$ baryonic transition [30], angular analysis of $B_{s} \rightarrow \phi \mu^{+} \mu^{-}$ [14] and $\Lambda_{b} \rightarrow \Lambda \mu^{+} \mu^{-}$decays [31], the first search for $B^{+} \rightarrow K^{+} \tau^{+} \tau^{-}$[32], and many more that cannot be detailed in this report.

\subsection{Radiative decays}

The inclusive radiative $\bar{B} \rightarrow X_{s, d} \gamma$ decays have been the subject of intense studies over the last few decades. On the theoretical side the dominant contributions to these decays are described by a local operator product expansion (OPE) known at next-next-to-leading-order [33] (with the exception of the exact $m_{c}$ dependence of the $\mathscr{O}_{1,2}-\mathscr{O}_{7}$ interference $\left.[34,35]\right)$. Subdominant corrections appear at the power-suppressed level $\left(\sim \Lambda_{\mathrm{QCD}} / m_{b}\right)$ and can be divided into local and non-local. The former are known through $O\left(\alpha_{s} \Lambda_{\mathrm{QCD}}^{2} / m_{b}^{2}\right)$ [36] and $O\left(\Lambda_{\mathrm{QCD}}^{5} / m_{b}^{5}\right)$ [37], albeit with poor knowledge of the higher-power matrix elements. The latter lead to resolved photon contributions in which the photon emission is a long-distance rescattering effect (e.g. $b \rightarrow s c \bar{c} \rightarrow s g \gamma$ ) [38]. Calculations of these effects are under very poor theoretical control and are essentially used to set an upper limit of about $5 \%$ on the their possible size; this is the last single source of uncertainty on the theoretical prediction for these branching ratios. The current theoretical predictions are [33]: $\operatorname{BR}\left(\bar{B} \rightarrow X_{s} \gamma\right)_{E_{\gamma}>1.6 \mathrm{GeV}}^{\mathrm{SM}}=(3.36 \pm 0.23) \times 10^{-4}$ and $\mathrm{BR}\left(\bar{B} \rightarrow X_{d} \gamma\right)_{E_{\gamma}>1.6 \mathrm{GeV}}^{\mathrm{SM}}=\left(1.73_{-0.22}^{+0.12}\right) \times 10^{-5}$. 
Recently Belle presented an updated measurement using their full $711 \mathrm{fb}^{-1}$ data set [39]: $\operatorname{BR}(\bar{B} \rightarrow$ $\left.X_{s} \gamma\right)_{E_{\gamma}>1.6 \mathrm{GeV}}^{\exp }=\left(3.12 \pm 0.10_{\text {stat }} \pm 0.19_{\text {syst }} \pm 0.08_{\text {model }}\right) \times 10^{-4}$. The accuracy of this single measurement is identical to that of the previous world average. The uncertainty on this measurement is already dominated by systematics, most of which can be reduced by further studies with a larger data set. A total uncertainty of $3.2 \%$ is reachable with $50 \mathrm{ab}^{-1}$ at Belle II.

The situation is radically different for exclusive modes, where only the magnetic-moment operator contribution is described in terms of a local OPE, requiring the tensor $B \rightarrow\left(K^{*}, \rho, \ldots\right)$ and $B_{s} \rightarrow\left(\phi, \bar{K}^{*}, \ldots\right)$ form factors. Contributions of other operators can be calculated within the QCD factorization approach up to non-local power corrections. The latter introduce very sizable uncertainties that can be somewhat reduced by considering ratios and asymmetries like $\mathrm{BR}\left(B \rightarrow K^{*} \gamma\right) / \mathrm{BR}\left(B_{s} \rightarrow \phi \gamma\right)$ and the $B \rightarrow\left(K^{*}, \rho\right) \gamma$ isospin asymmetries, which are predicted with uncertainties of $23 \%$ and $54 \%$, respectively [40]. Other observables, like the time-dependent $C P$ asymmetry measured in $B^{0}, \bar{B}^{0} \rightarrow f_{C P} \gamma$ are expected to be vanishingly small in the SM due to the chiral nature of weak interactions and offer sensitive tests of non-standard right-handed currents. In this context, a result for the time-dependent $C P$ asymmetry in the $K_{S}^{0} \rho \gamma$ final state has been recently obtained by BaBar [41], resulting in $S_{K_{S}^{0} \rho \gamma}=-0.18 \pm 0.32_{-0.05}^{+0.06}$, which is compatible with the $\mathrm{SM}$ expectation $(S \sim 0.02)$. The time-dependent decay rate of untagged $B_{S} \rightarrow \phi \gamma$ is also sensitive to the photon polarization via the coefficient of the sinh term, $A^{\Delta}$ [42]. LHCb first measured this observable, obtaining $A^{\Delta}=-0.98_{-0.52-0.20}^{+0.46+0.23}$ [43], which is consistent with the SM prediction from [42], $A^{\Delta}=0.0047_{-0.025}^{+0.029}$.

\subsection{Global analysis of $b \rightarrow s$ decays}

Global fits to $b \rightarrow s$ anomalies involve the combination of several exclusive and inclusive $b \rightarrow s \gamma$ and $b \rightarrow s \ell \ell$ transitions: $B \rightarrow\left(K^{*}, X_{s}\right) \gamma$ (sensitive to $C_{7}^{(\prime)}$ ), $B_{s} \rightarrow \ell \ell$ (sensitive to $C_{10}^{(\prime)}$ ), $B \rightarrow$ $\left(K, K^{*}, X_{s}\right) \ell \ell$, and $B_{s} \rightarrow \phi \ell \ell$ (sensitive to $C_{7,9,10}^{(\prime)}$ ). These studies revolve around the experimental tensions in exclusive $b \rightarrow s \ell \ell$ decays and depend critically on theoretical systematic uncertainties.

The decays $B \rightarrow\left(K, K^{*}\right) \ell \ell$ and $B_{s} \rightarrow \phi \ell \ell$ are described using Soft-Collinear Effective Theory (SCET) $[44,45]$ at low $q^{2}$, where the final-state hadron has large energy, and by a local OPE [46, 47] at high $q^{2}$, where the final-state hadron is almost at rest.

At low $q^{2}$, the leading contributions to the amplitudes are expressed in terms of heavy-tolight $B_{q}$ form factors and meson light-cone distribution amplitudes. Unfortunately, there is no widespread agreement on the actual size of the sub-leading corrections, which are expected to scale as $\Lambda_{\mathrm{QCD}} / m_{b}$. For the calculation of branching ratios, it is always possible to use full QCD form factors, thus confining power corrections to the matrix elements of the operators $\mathscr{O}_{1,2}$ whose contribution to the total amplitude is subdominant (see for instance the $B \rightarrow K \ell \ell$ analysis presented in Ref. [48]). The main problem resides in the calculation of asymmetries and ratios (e.g. $P_{5}^{\prime}$ ) for which the leading dependence on the form factors cancels as long as the form factors themselves are calculated within SCET; this introduces additional power corrections, about the size of which there is currently no definite agreement $[49,50,51,52]$. At high $q^{2}$, the power corrections are local and thus under better control. Unfortunately, resonant charmonium contributions $\left(B \rightarrow K^{(*)} \psi_{c c} \rightarrow\right.$ $K^{(*)} \ell \ell$ introduce potential violations of quark-hadron duality that are difficult to estimate [53,54].

The form factors are the most important non-perturbative inputs to these calculations and are accessible using lattice QCD and Light-Cone QCD Sum Rules (LCSR). Lattice QCD offers a first- 

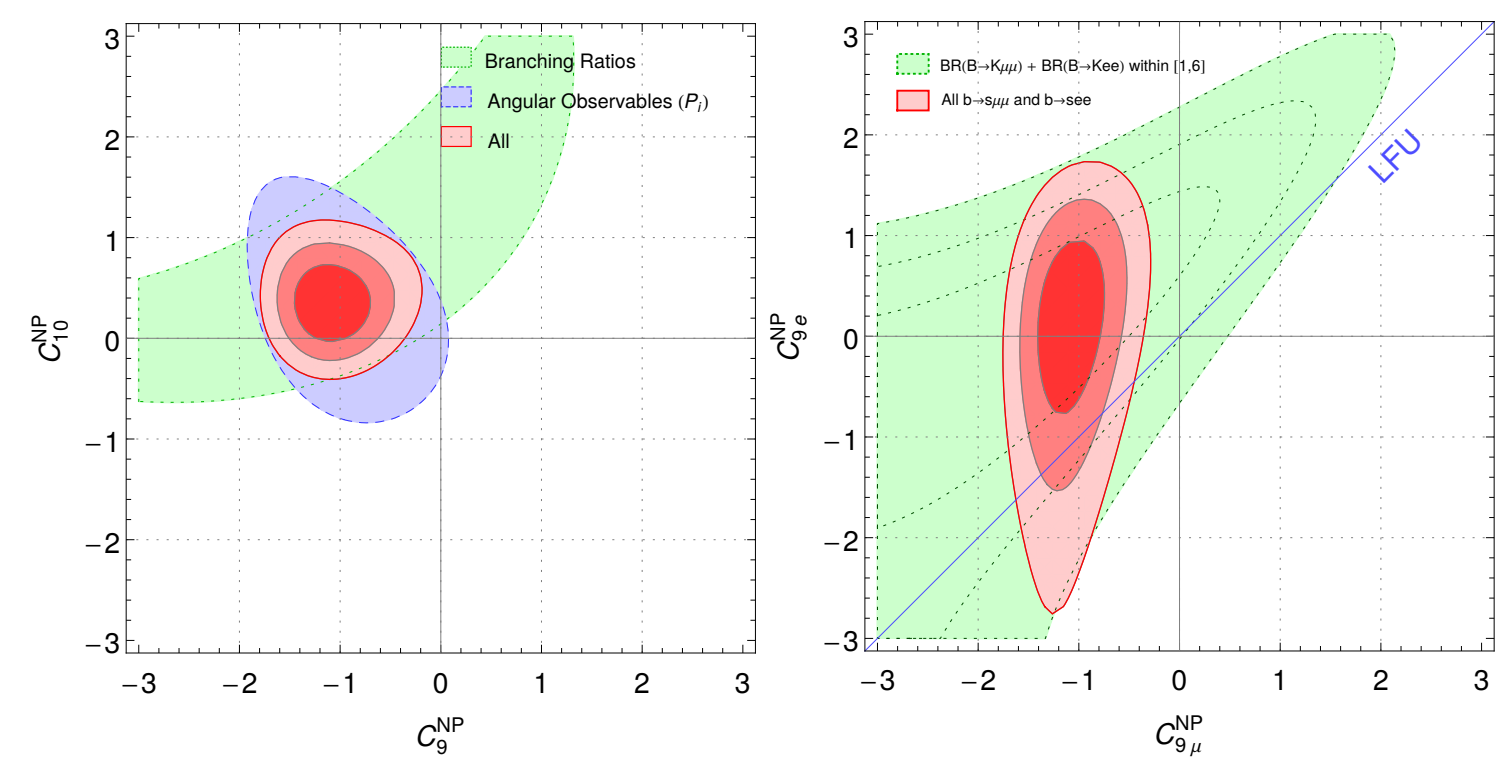

Figure 3: Global fits of $b \rightarrow s \ell \ell$ anomalies in the $\left(C_{9}^{\mathrm{NP}}, C_{10}^{\mathrm{NP}}\right)$ (left) and $\left(C_{9 \mu}^{\mathrm{NP}}, C_{9 e}\right)^{\mathrm{NP}}$ (right) planes. The plots are taken from Ref. [49].

principle calculation in which all sources of uncertainty can be systematically taken into account, but, for technical reasons, allows access to the form factors only at relatively large momentum transfer $\left(q^{2}\right)$. The LCSR approach, on the other hand, requires the final-state mesons to have large energy, implying small $q^{2}$. For these reasons it is common to see analyses using lattice QCD at low recoil and LCSR at high recoil. Recently, very high quality calculations of the three form factors $f_{+, 0, T}\left(q^{2}\right)$ for the $B \rightarrow \pi[55,56,57], B_{S} \rightarrow K[58,55]$, and $B \rightarrow K[59,60,61]$ channels have been performed. Decays into vector mesons are considerably more complex because there are seven independent form factors for each channel and the vector mesons undergo strong decays. A first complete study of $B \rightarrow K^{*}$ and $B_{s} \rightarrow\left(\phi, K^{*}\right)$ was presented in Ref. [62]. Bottom baryon form factors have been also investigated in the $\Lambda_{b} \rightarrow\left(p, \Lambda_{c}\right)$ [63] and $\Lambda_{b} \rightarrow \Lambda$ [31] channels. Calculations of $B \rightarrow\left(K^{*}, \rho, \omega\right)$ and $B_{s} \rightarrow\left(\phi, K^{*}\right)$ in the LCSR approach are presented in Ref. [64].

In the two panels of Fig. 3, taken from Ref. [49], we show the results of global fits in the $\left(C_{9}^{\mathrm{NP}}, C_{10}^{\mathrm{NP}}\right)$ plane assuming lepton flavour universality and in the $\left(C_{9 \mu}^{\mathrm{NP}}, C_{9 e}^{\mathrm{NP}}\right)$ plane after the inclusion of constraints from $R_{K}=\mathrm{BR}(B \rightarrow K \mu \mu) / \mathrm{BR}(B \rightarrow K e e)$ (see Ref. [11] for a review of the theoretical uncertainties on $R_{K}$ ).

New physics in $B \rightarrow K^{(*)} \ell \ell$ decays can be tested in the corresponding inclusive mode $B \rightarrow X_{s} \ell \ell$ for which power corrections are under much better theoretical control (see Ref. [65] for a theoretical review). Issues related to the calculation of the $X_{s}$ invariant-mass spectrum, which is relevant in order to asses the impact of required experimental cuts on $m_{X_{s}}$, have been studied within a Fermi motion model [66] and SCET [67, 68]. Current theoretical predictions have uncertainties that are at the level of the experimental accuracy achievable by Belle II with $50 \mathrm{fb}^{-1}$ of integrated luminosity [69]. 


\section{Rare $D$ decays}

Rare decays of $D$ mesons test for NP in FCNC transitions involving up-type quarks. In the SM, the short-distance contributions are strongly GIM suppressed and long-distance effects are expected to dominate. This makes it very challenging to disentangle potential NP contributions from the SM background. Correlations between different measurements can help to discriminate NP effects.

As an example, in the $\mathrm{SM}$, short-distance physics contributes about $10^{-18}$ to $\mathrm{BR}(D \rightarrow \mu \mu)$, while the total BR is dominated by the long-distance amplitude mediated by two photons [70]. Specifically, $\operatorname{BR}(D \rightarrow \mu \mu)$ is expected to be about $2.7 \times 10^{-5} \times \operatorname{BR}(D \rightarrow \gamma \gamma)$, or about $10^{-13}$, with $\operatorname{BR}(D \rightarrow \gamma \gamma)$ expected to be $\leq 10^{-8}$. In various NP models, $\operatorname{BR}(D \rightarrow \mu \mu)$ is correlated with the value of the mixing parameter $x_{D}$. For example, in theories with heavy vector-like quarks, FCNC interactions are generated in the left-handed up-quark sector [71], leading to the prediction $\mathrm{BR}(D \rightarrow \mu \mu) \approx 4.3 \times 10^{-9} x_{D} \leq 4.3 \times 10^{-11}$.

For the $D \rightarrow \gamma \gamma$ decay, the short-distance, EM-penguin contribution to the BR is $3 \times 10^{-11}$ [72], while the long-distance contribution from vector-meson dominance is $(1-3) \times 10^{-8}$ [73]. A NP contribution from the MSSM (due to gluino exchange via $c \rightarrow u \gamma$ transitions) would give $6 \times 10^{-6}$ [74]. The most stringent limit, recently published by Belle, was obtained from $D^{*+} \rightarrow D^{0} \pi^{+}$: $\operatorname{BR}(D \rightarrow \gamma \gamma)<8.5 \times 10^{-7}(90 \% \mathrm{CL})$ [75]. Note that this provides an experimental upper bound for the expected value of the long-distance $\mathrm{SM}$ contribution to $\mathrm{BR}(D \rightarrow \mu \mu)$.

For $D \rightarrow P \ell \ell$ decays, the GIM suppression is very effective and the SM rates are expected to be small and dominated by long-distance effects, including production of the $\rho / \omega$ and $\phi$ resonances, which contribute to the BR at the level of $10^{-6}$. The non-resonant contribution to the BR is at the level of $10^{-12}[76,77]$. New physics could contribute to the BR at the level of $10^{-9}$ in the case of heavy vector-like quarks in the up sector, or as much as a few $\times 10^{-6}$ in the case of the MSSM with $R$-parity violation. The effects could be obscured by the resonant contribution, but measurement of the decay spectrum $d \mathrm{BR} / d q^{2}$ could help to isolate NP contributions [76]. BESIII has recent preliminary results for the decays $D^{+} \rightarrow K^{ \pm} e^{+} e^{\mp}$ and $D^{+} \rightarrow \pi^{ \pm} e^{+} e^{\mp}$, obtained with single-tagged events from $\psi(3770)$ decays. The observation of the lepton-number-violating decays $D^{+} \rightarrow K^{-}\left(\pi^{-}\right) e^{+} e^{+}$would indicate the existence of a Majorana neutrino mass term [78]. The limits from BES-III on the BRs for $K^{-} e^{+} e^{+}\left(6 \times 10^{-7}\right)$ and $\pi^{+} e^{+} e^{-}\left(3 \times 10^{-7}\right)$ are the most stringent obtained to date for these lepton-number-violating and -conserving decays, respectively. BES-III also presented a new limit on $D^{+} \rightarrow D^{0} e^{+} v_{e}$ at this conference: $\mathrm{BR}<8.7 \times 10^{-5}(90 \%$ CL), obtained via a double-tag technique with the $D^{0}$ decaying to $K \pi, K \pi \pi^{0}$, or $K \pi \pi \pi$. The expected BR in the SU(3) limit is $2.78 \times 10^{-13}$ [79].

The short-distance contributions for $D^{*}\left(B^{*}\right) \rightarrow \ell \ell$ decays [80] are expected to be larger than the long-distance contributions. Since the SM BRs are on the order of $10^{-19}$ they cannot be measured directly. Various NP models could increase the rate by orders of magnitude. For instance, in the presence of a flavour-changing $Z^{\prime}$ coupling to left-handed quark currents, the BR could be as high as $2.5 \times 10^{-11}$. It may be possible to measure the resonant production of $D^{*}(2007)$ and subsequent strong or electromagnetic decay to $D^{0} \pi^{0}$ or $D^{0} \gamma$ in $e^{+} e^{-}$collisions at $\sqrt{s}=m_{D^{*}}$, for example, at BEPC-II with the BES-III detector, or possibly at VEPP-2000 with an upgrade to increase the machine energy above $2 \mathrm{GeV}$. 
$D$ (or $B$ ) decays with missing energy could be a dark-matter signature [81]. The $D \rightarrow v \bar{v}$ decay is helicity suppressed and has an SM BR of $1.1 \times 10^{-30}$. Adding a final-state photon overcomes the helicity suppression, but the SM BR is still $3.96 \times 10^{-14}$. In a minimal scalar dark matter model, the BR for $D \rightarrow S S$ (with $S$ a scalar dark matter particle) could be on the order of $10^{-14}$. Belle has recently published the first limit on $\operatorname{BR}\left(D^{0} \rightarrow\right.$ invisible) obtained using the charm-tag method, in which $e^{+} e^{-} \rightarrow D_{\text {tag }}^{(*)} X_{\text {frag }} \bar{D}_{\text {sig }}^{*-}, D_{\text {tag }}^{(*)}$ and $X_{\text {frag }}$ are reconstructed from a variety of modes, and the $\pi^{-}$ from $\bar{D}_{\text {sig }}^{*-} \rightarrow \bar{D}^{0} \pi^{-}$is reconstructed as well. The result is $\mathrm{BR}<8.8 \times 10^{-5}$ (90\% CL) [82].

\section{Rare $K$ decays}

The SM calculation of the BRs for the $K \rightarrow \pi v \bar{v}$ decays is particularly clean because the loop amplitudes are dominated by the top-quark contributions, the hadronic matrix element can be obtained from the precisely known $K_{e 3}$ rate, and there are no long-distance contributions from processes with intermediate photons. In the $\mathrm{SM}, \operatorname{BR}\left(K^{+} \rightarrow \pi^{+} v \bar{v}\right)=(8.4 \pm 1.0) \times 10^{-11}$ and $\operatorname{BR}\left(K_{L}^{0} \rightarrow \pi^{0} v \bar{v}\right)=(3.4 \pm 0.6) \times 10^{-11}$ [83]. The dominant uncertainties are from $V_{c b}, V_{u b}$ and $\gamma$; the underlying theoretical uncertainties are just 0.30 and $0.05 \times 10^{-11}$, respectively. If both BRs are measured and one or both is different from its SM value, it may be possible to characterize the NP responsible (see e.g. [84]).

Seven candidate $K^{+} \rightarrow \pi^{+} v \bar{v}$ events have been seen by the Brookhaven experiment E787 and its successor, E949, giving $\mathrm{BR}\left(K^{+} \rightarrow \pi^{+} v \bar{v}\right)=1.73_{-1.05}^{+1.15} \times 10^{-10}$ [85]. The goal of the NA62 experiment [4] is to measure $\mathrm{BR}\left(K^{+} \rightarrow \pi^{+} v \bar{v}\right)$ with a precision of about $10 \%$. NA62 makes use of a $750 \mathrm{MHz}, 75-\mathrm{GeV}$ positive secondary beam from the CERN SPS, providing $5 \mathrm{MHz}$ of $K^{+}$decays in a 60-m fiducial volume. The full beam rate is tracked through three stations of silicon pixel detectors, and kaons in the beam are identified by a differential Cerenkov counter. Secondaries are tracked through four ultra-light straw tube stations operated in vacuum. Precise reconstruction of the missing mass at the decay vertex provides rejection against the dominant decays $K^{+} \rightarrow \mu^{+} v$ and $K^{+} \rightarrow \pi^{+} \pi^{0}$. Photons from $\pi^{0}$ are vetoed by EM calorimeters with hermetic coverage for angles below $50 \mathrm{mrad}$, including the NA48 liquid-krypton calorimeter ( $\mathrm{LKr})$. Muon vetoes and hadron calorimeters downstream of the $\mathrm{LKr}$ provide $\mu / \pi$ separation, while a RICH provides additional particle identification for secondaries and helps with the precision time measurement needed for accurate $K^{+}-\pi^{+}$association. NA62 is currently taking data and collected $10^{12} K^{+}$decays in 2016. The experiment aims to collect $10^{13} K^{+}$decays to observe $\sim 100$ signal events by the end of 2018 . NA62 can collect several triggers simultaneously to address a broad physics portfolio, and plans to explore the dark sector in runs after the second long shutdown of the LHC.

The decay $K_{L}^{0} \rightarrow \pi^{0} v \bar{v}$ has never been observed. Model-independent arguments [86] and the measured value of $\operatorname{BR}\left(K^{+} \rightarrow \pi^{+} v \bar{v}\right)$ lead to the Grossman-Nir upper limit $\operatorname{BR}\left(K_{L}^{0} \rightarrow \pi^{0} v \bar{v}\right)<$ $1.4 \times 10^{-9}$. The KOTO experiment at J-PARC (Tokai, Japan) is making steady progress at pushing down the direct limit. The experiment makes use of a tightly collimated neutral beam (average momentum $2 \mathrm{GeV}$ ) and compact, hermetic detector. From a brief pilot run in 2013, KOTO obtained the limit $\mathrm{BR}\left(K_{L}^{0} \rightarrow \pi^{0} v \bar{v}\right)<5.1 \times 10^{-8}$ (90\% CL) [6]. The experiment ran for many months in 2015-2016, with various improvements to reduce the background. From the preliminary analysis of $10 \%$ of the data collected in 2015, KOTO has reached a single-event BR sensitivity of $5.9 \times 10^{-9}$, with background levels still under evaluation. With the entire 2015-2016 data set, KOTO should 
be able to push the single-event sensitivity to below the Grossman-Nir bound. By the end of 2015 , the beam power reached $42 \mathrm{~kW}$; it is expected to increase to $100 \mathrm{~kW}$ by 2018 . A series of upgrades to the experiment are also in progress, including an additional layer for the barrel calorimeter (tested in 2016) and front/back readout for the forward CsI calorimeter to provide additional neutron rejection. The experiment expects to reach single-event sensitivity at the level of the SM BR by about 2021.

Numerous other rare kaon decays are of interest besides $K \rightarrow \pi v \bar{v}$. Since the $C P$-violating phase of the $s \rightarrow d \ell^{+} \ell^{-}$transition is poorly determined, there is room for NP to manifest itself in $K \rightarrow \pi \ell^{+} \ell^{-}$and $K \rightarrow \ell^{+} \ell^{-}$decays, although there are complications from the long-distance contributions [87]. For example, $K_{L} \rightarrow \mu^{+} \mu^{-}$is dominated by the long-distance component arising via $K_{L} \rightarrow \gamma \gamma$. On the other hand, about one-third of the $K_{S} \rightarrow \mu^{+} \mu^{-}$width (BR $\sim 5 \times 10^{-12}$ ) is from the $C P$-violating short-distance amplitude [88]. The recent limit from $\mathrm{LHCb}, \mathrm{BR}\left(K_{S} \rightarrow\right.$ $\left.\mu^{+} \mu^{-}\right)<5.8 \times 10^{-9}\left(90 \%\right.$ CL) [89], demonstrates LHCb's capability to measure $K_{S}$ decays to muons. LHCb may also be able to measure $\operatorname{BR}\left(K_{S} \rightarrow \pi^{0} \mu^{+} \mu^{-}\right)$, which helps to isolate the shortdistance component of $K_{L} \rightarrow \pi^{0} \mu^{+} \mu^{-}$by pinning down the contribution from indirect $C P$ violation. The $K^{+} \rightarrow \pi^{+} \ell^{+} \ell^{-}$decays may show signs of lepton-flavour universality violation, in analogy to the $R_{K}$ and $P_{5}^{\prime}$ anomalies observed in the $B$ system. NA62 may have the statistics to considerably improve on NA48/2 results for the form-factor slopes for these decays. High-statistics measurements of the Dalitz plots for the $K \rightarrow \pi \pi \gamma$ and $K \rightarrow \pi \pi e e$ decays would allow the innerbremsstrahlung and direct-emission contributions to be isolated, enabling searches for $C P$-violating amplitudes in the latter case. Finally, NA62 and other experiments measuring $\pi^{ \pm}$and $K^{ \pm}$decays could also look for time-dependent anisotropies in the lepton momentum distribution in $P_{\mu 2}$, which would be observable if Lorentz invariance were violated and the fundamental symmetry group were instead the $\operatorname{SIM}(2)$ subgroup, implying the existence of a preferred direction in spacetime [90].

\section{Charged lepton flavour violation}

In the SM, charged lepton flavour violating decays are highly suppressed due to the smallness of neutrino masses. The observation of such a decay would therefore be an unambiguous sign of physics beyond the SM.

Lepton flavour violating decays of muons are searched for at dedicated facilities. The MEG experiment at PSI has improved the limit on the $\mu^{+} \rightarrow e^{+} \gamma$ decay by a factor 30 with respect to the previous experiment using their final data set: $\operatorname{BR}\left(\mu^{+} \rightarrow e^{+} \gamma\right)<4.2 \times 10^{-13}$ at $90 \%$ CL [91]. The experiment has been upgraded to reach a sensitivity of $4 \times 10^{-14}$ and will have a first engineering run in 2017. The $\mu^{+} \rightarrow e^{+} e^{-} e^{+}$decay has been searched for previously by the SINDRUM experiment reaching, $1.0 \times 10^{-12}$. The Mu3e experiment at PSI aims at reaching a sensitivity of $10^{-15}$ as a first step in 2018-2020, and $10^{-16}$ in a second step. Muon-to-electron conversion will be searched for by COMET at J-PARC and Mu2e at Fermilab. They both expect to reach a single event sensitivity of $\sim 2 \times 10^{-17}$ using aluminium targets in the years 2020 .

Lepton flavour violation is also searched for in $\tau$ and $B$ decays at the $B$ factories and LHC experiments. A few new results have appeared in the last couple of years. The majority of the limits for $\tau$ decays are dominated by the Belle collaboration, reaching limits in the range $10^{-8}$ to $2 \times 10^{-7}$, except for $\tau^{+} \rightarrow e^{+} / \mu^{+} \gamma$, where BaBar has the most stringent limits, and $\tau \rightarrow p \mu^{+} \mu^{-}$, 
which has only been searched for by LHCb. Regarding the $B$ decays, BaBar has obtained the best limits in most cases, spanning a range from a few $\times 10^{-8}$ for the $e$ and $\mu$ channels to a few $\times 10^{-5}$ for channels involving $\tau$ leptons. LHCb has obtained the best limits for the $B^{0} \rightarrow e^{ \pm} \mu^{\mp}$ channel, reaching a limit at $2.8 \times 10^{-9}(90 \% \mathrm{CL})$ with $1 \mathrm{fb}^{-1}$ of Run 1 data. For a complete picture of $\tau$ and $B$ LFV results, see Ref. [92].

\section{Summary}

While generally speaking, present results on rare $B$ decays are in agreement with SM predictions, measurements of some observables, notably $R_{K}$ and $P_{5}^{\prime}$, show intriguing hints of significant deviation. It is very encouraging to see the excellent reach of LHCb with only Run 1 data, as well as the improvements in the results from the $B$-factory experiments, which are continuously refining their analysis techniques to obtain more sensitive tests of the SM. This year's CKM conference has also seen the first results on rare $B$ decays to $\tau$ leptons, which are interesting in view of the hints of lepton flavour universality violation in decays to muons. Within the next few years, new results from LHCb, ATLAS, and CMS Run 2 data will be supplemented by the arrival of the first results from Belle II and from the kaon experiments KOTO and NA62, as well as by results from the next generation of charged lepton flavour violation experiments. The prospects are therefore excellent for rare decays to continue to place new and increasingly tight constraints on the Standard Model.

\section{References}

[1] N. Cabibbo, Phys. Rev. Lett. 10, 531 (1963). doi:10.1103/PhysRevLett.10.531

[2] M. Kobayashi and T. Maskawa, Prog. Theor. Phys. 49, 652 (1973). doi:10.1143/PTP.49.652

[3] Talk by R. Itoh at "Sixth Workshop on Theory, Phenomenology and Experiments in Flavour Physics FPCapri2016", Capri, Italy, 11-13 June 2016.

[4] E. Cortina Gil et al. [NA62 Collaboration], arXiv:1703.08501 [physics.ins-det].

[5] T. K. Komatsubara, Prog. Part. Nucl. Phys. 67, 995 (2012) doi:10.1016/j.ppnp.2012.04.001 [arXiv:1203.6437 [hep-ex]].

[6] J. K. Ahn et al., PTEP 2017, no. 2, $021 \mathrm{C01}$ (2017) doi:10.1093/ptep/ptx001 [arXiv:1609.03637 [hep-ex]].

[7] C. Bobeth, M. Gorbahn, T. Hermann, M. Misiak, E. Stamou and M. Steinhauser, Phys. Rev. Lett. 112 (2014) 101801 doi:10.1103/PhysRevLett.112.101801 [arXiv:1311.0903 [hep-ph]].

[8] M. Aaboud et al. [ATLAS Collaboration], Eur. Phys. J. C 76 (2016) no.9, 513 doi:10.1140/epjc/s10052-016-4338-8 [arXiv:1604.04263 [hep-ex]].

[9] K. De Bruyn [LHCb Collaboration], LHCb-CONF-2016-011, CERN-LHCb-CONF-2016-011.

[10] R. Aaij et al. [LHCb Collaboration], Phys. Rev. Lett. 113 (2014) 151601 doi:10.1103/PhysRevLett.113.151601 [arXiv:1406.6482 [hep-ex]].

[11] M. Bordone, G. Isidori and A. Pattori, Eur. Phys. J. C 76 (2016) no.8, 440 doi:10.1140/epjc/s10052-016-4274-7 [arXiv:1605.07633 [hep-ph]]. 
[12] R. Aaij et al. [LHCb Collaboration], JHEP 1406 (2014) 133 doi:10.1007/JHEP06(2014)133 [arXiv:1403.8044 [hep-ex]].

[13] R. Aaij et al. [LHCb Collaboration], JHEP 1506 (2015) 115 doi:10.1007/JHEP06(2015)115 [arXiv:1503.07138 [hep-ex]].

[14] R. Aaij et al. [LHCb Collaboration], JHEP 1509 (2015) 179 doi:10.1007/JHEP09(2015)179 [arXiv:1506.08777 [hep-ex]].

[15] R. Aaij et al. [LHCb Collaboration], JHEP 1611 (2016) 047 doi:10.1007/JHEP11(2016)047 [arXiv:1606.04731 [hep-ex]].

[16] S. Descotes-Genon, L. Hofer, J. Matias and J. Virto, JHEP 1412 (2014) 125 doi:10.1007/JHEP12(2014)125 [arXiv:1407.8526 [hep-ph]].

[17] J. P. Lees et al. [BaBar Collaboration], Phys. Rev. D 93 (2016) no.5, 052015 doi:10.1103/PhysRevD.93.052015 [arXiv:1508.07960 [hep-ex]].

[18] V. Khachatryan et al. [CMS Collaboration], Phys. Lett. B 753 (2016) 424 doi:10.1016/j.physletb.2015.12.020 [arXiv:1507.08126 [hep-ex]].

[19] R. Aaij et al. [LHCb Collaboration], JHEP 1602 (2016) 104 doi:10.1007/JHEP02(2016)104 [arXiv:1512.04442 [hep-ex]].

[20] S. Wehle et al. [Belle Collaboration], Phys. Rev. Lett. 118, no. 11, 111801 (2017) doi:10.1103/PhysRevLett.118.111801 [arXiv:1612.05014 [hep-ex]].

[21] B. Capdevila, S. Descotes-Genon, J. Matias and J. Virto, JHEP 1610 (2016) 075 doi:10.1007/JHEP10(2016)075 [arXiv:1605.03156 [hep-ph]].

[22] D. Bhatia, S. Chakraborty and A. Dighe, JHEP 1703, 117 (2017) doi:10.1007/JHEP03(2017)117 [arXiv:1701.05825 [hep-ph]].

[23] C. Hambrock, A. Khodjamirian and A. Rusov, Phys. Rev. D 92 (2015) no.7, 074020 doi:10.1103/PhysRevD.92.074020 [arXiv:1506.07760 [hep-ph]].

[24] A. J. Buras, J. Girrbach-Noe, C. Niehoff and D. M. Straub, JHEP 1502 (2015) 184 doi:10.1007/JHEP02(2015)184 [arXiv:1409.4557 [hep-ph]].

[25] O. Lutz et al. [Belle Collaboration], Phys. Rev. D 87 (2013) no.11, 111103 doi:10.1103/PhysRevD.87.111103 [arXiv:1303.3719 [hep-ex]].

[26] J. P. Lees et al. [BaBar Collaboration], Phys. Rev. D 87 (2013) no.11, 112005 doi:10.1103/PhysRevD.87.112005 [arXiv:1303.7465 [hep-ex]].

[27] P. del Amo Sanchez et al. [BaBar Collaboration], Phys. Rev. D 82 (2010) 112002 doi:10.1103/PhysRevD.82.112002 [arXiv:1009.1529 [hep-ex]].

[28] J. Grygier et al. [Belle Collaboration], arXiv:1702.03224 [hep-ex].

[29] A. Schwartz, N. G. Deshpande and J. Urheim, Phys. Rev. D 44, 291 (1991). doi:10.1103/PhysRevD.44.291

[30] R. Aaij et al. [LHCb Collaboration], arXiv:1701.08705 [hep-ex].

[31] W. Detmold and S. Meinel, Phys. Rev. D 93 (2016) no.7, 074501 doi:10.1103/PhysRevD.93.074501 [arXiv:1602.01399 [hep-lat]].

[32] J. P. Lees et al. [BaBar Collaboration], Phys. Rev. Lett. 118 (2017) no.3, 031802 doi:10.1103/PhysRevLett.118.031802 [arXiv:1605.09637 [hep-ex]]. 
[33] M. Misiak et al., Phys. Rev. Lett. 114, no. 22, 221801 (2015) doi:10.1103/PhysRevLett.114.221801 [arXiv:1503.01789 [hep-ph]].

[34] M. Czakon, P. Fiedler, T. Huber, M. Misiak, T. Schutzmeier and M. Steinhauser, JHEP 1504, 168 (2015) doi:10.1007/JHEP04(2015)168 [arXiv:1503.01791 [hep-ph]].

[35] M. Misiak, A. Rehman and M. Steinhauser, arXiv:1702.07674 [hep-ph].

[36] T. Ewerth, P. Gambino and S. Nandi, Nucl. Phys. B 830, 278 (2010) doi:10.1016/j.nuclphysb.2009.12.035 [arXiv:0911.2175 [hep-ph]].

[37] P. Gambino, K. J. Healey and S. Turczyk, Phys. Lett. B 763, 60 (2016) doi:10.1016/j.physletb.2016.10.023 [arXiv:1606.06174 [hep-ph]].

[38] M. Benzke, S. J. Lee, M. Neubert and G. Paz, JHEP 1008, 099 (2010) doi:10.1007/JHEP08(2010)099 [arXiv:1003.5012 [hep-ph]].

[39] A. Abdesselam et al. [Belle Collaboration], arXiv:1608.02344 [hep-ex].

[40] J. Lyon and R. Zwicky, Phys. Rev. D 88, no. 9, 094004 (2013) doi:10.1103/PhysRevD.88.094004 [arXiv:1305.4797 [hep-ph]].

[41] P. del Amo Sanchez et al. [BaBar Collaboration], Phys. Rev. D 93, no. 5, 052013 (2016) doi:10.1103/PhysRevD.93.052013 [arXiv:1512.03579 [hep-ex]].

[42] F. Muheim, Y. Xie and R. Zwicky, Phys. Lett. B 664, 174 (2008) doi:10.1016/j.physletb.2008.05.032 [arXiv:0802.0876 [hep-ph]].

[43] R. Aaij et al. [LHCb Collaboration], Phys. Rev. Lett. 118, no. 2, 021801 (2017) Addendum: [Phys. Rev. Lett. 118, no. 10, 109901 (2017)] doi:10.1103/PhysRevLett.118.021801, 10.1103/PhysRevLett.118.109901 [arXiv:1609.02032 [hep-ex]].

[44] C. W. Bauer, S. Fleming, D. Pirjol and I. W. Stewart, Phys. Rev. D 63, 114020 (2001) doi:10.1103/PhysRevD.63.114020 [hep-ph/0011336].

[45] M. Beneke, T. Feldmann and D. Seidel, Nucl. Phys. B 612, 25 (2001) doi:10.1016/S0550-3213(01)00366-2 [hep-ph/0106067].

[46] B. Grinstein and D. Pirjol, Phys. Rev. D 70, 114005 (2004) doi:10.1103/PhysRevD.70.114005 [hep-ph/0404250].

[47] C. Bobeth, G. Hiller and D. van Dyk, JHEP 1007, 098 (2010) doi:10.1007/JHEP07(2010)098 [arXiv:1006.5013 [hep-ph]].

[48] D. Du, A. X. El-Khadra, S. Gottlieb, A. S. Kronfeld, J. Laiho, E. Lunghi, R. S. Van de Water and R. Zhou, Phys. Rev. D 93, no. 3, 034005 (2016) doi:10.1103/PhysRevD.93.034005 [arXiv:1510.02349 [hep-ph]].

[49] S. Descotes-Genon, L. Hofer, J. Matias and J. Virto, JHEP 1606, 092 (2016) doi:10.1007/JHEP06(2016)092 [arXiv:1510.04239 [hep-ph]].

[50] S. Jäger and J. Martin Camalich, JHEP 1305, 043 (2013) doi:10.1007/JHEP05(2013)043 [arXiv:1212.2263 [hep-ph]].

[51] S. Jäger and J. Martin Camalich, Phys. Rev. D 93, no. 1, 014028 (2016) doi:10.1103/PhysRevD.93.014028 [arXiv:1412.3183 [hep-ph]].

[52] M. Ciuchini, M. Fedele, E. Franco, S. Mishima, A. Paul, L. Silvestrini and M. Valli, JHEP 1606, 116 (2016) doi:10.1007/JHEP06(2016)116 [arXiv:1512.07157 [hep-ph]]. 
[53] M. Beylich, G. Buchalla and T. Feldmann, Eur. Phys. J. C 71, 1635 (2011) doi:10.1140/epjc/s10052-011-1635-0 [arXiv:1101.5118 [hep-ph]].

[54] J. Lyon and R. Zwicky, arXiv:1406.0566 [hep-ph].

[55] J. M. Flynn, T. Izubuchi, T. Kawanai, C. Lehner, A. Soni, R. S. Van de Water and O. Witzel, Phys. Rev. D 91, no. 7, 074510 (2015) doi:10.1103/PhysRevD.91.074510 [arXiv:1501.05373 [hep-lat]].

[56] J. A. Bailey et al. [Fermilab Lattice and MILC Collaborations], Phys. Rev. D 92, no. 1, 014024 (2015) doi:10.1103/PhysRevD.92.014024 [arXiv:1503.07839 [hep-lat]].

[57] J. A. Bailey et al. [Fermilab Lattice and MILC Collaborations], Phys. Rev. Lett. 115, no. 15, 152002 (2015) doi:10.1103/PhysRevLett.115.152002 [arXiv:1507.01618 [hep-ph]].

[58] C. M. Bouchard, G. P. Lepage, C. Monahan, H. Na and J. Shigemitsu, Phys. Rev. D 90, 054506 (2014) doi:10.1103/PhysRevD.90.054506 [arXiv:1406.2279 [hep-lat]].

[59] C. Bouchard et al. [HPQCD Collaboration], Phys. Rev. D 88, no. 5, 054509 (2013) Erratum: [Phys. Rev. D 88, no. 7, 079901 (2013)] doi:10.1103/PhysRevD.88.079901, 10.1103/PhysRevD.88.054509 [arXiv:1306.2384 [hep-lat]].

[60] C. Bouchard et al. [HPQCD Collaboration], Phys. Rev. Lett. 111, no. 16, 162002 (2013) Erratum: [Phys. Rev. Lett. 112, no. 14, 149902 (2014)] doi:10.1103/PhysRevLett.112.149902, 10.1103/PhysRevLett.111.162002 [arXiv:1306.0434 [hep-ph]].

[61] J. A. Bailey et al., Phys. Rev. D 93, no. 2, 025026 (2016) doi:10.1103/PhysRevD.93.025026 [arXiv:1509.06235 [hep-lat]].

[62] R. R. Horgan, Z. Liu, S. Meinel and M. Wingate, Phys. Rev. D 89, no. 9, 094501 (2014) doi:10.1103/PhysRevD.89.094501 [arXiv:1310.3722 [hep-lat]].

[63] W. Detmold, C. Lehner and S. Meinel, Phys. Rev. D 92, no. 3, 034503 (2015) doi:10.1103/PhysRevD.92.034503 [arXiv:1503.01421 [hep-lat]].

[64] A. Bharucha, D. M. Straub and R. Zwicky, JHEP 1608, 098 (2016) doi:10.1007/JHEP08(2016)098 [arXiv:1503.05534 [hep-ph]].

[65] T. Huber, T. Hurth and E. Lunghi, JHEP 1506, 176 (2015) doi:10.1007/JHEP06(2015)176 [arXiv: 1503.04849 [hep-ph]].

[66] A. Ali, G. Hiller, L. T. Handoko and T. Morozumi, Phys. Rev. D 55, 4105 (1997) doi:10.1103/PhysRevD.55.4105 [hep-ph/9609449].

[67] K. S. M. Lee, Z. Ligeti, I. W. Stewart and F. J. Tackmann, Phys. Rev. D 74, 011501 (2006) doi:10.1103/PhysRevD.74.011501 [hep-ph/0512191].

[68] M. Benzke, M. Fickinger, T. Hurth and S. Turczyk, to appear.

[69] T. Hurth, F. Mahmoudi and S. Neshatpour, JHEP 1412, 053 (2014) doi:10.1007/JHEP12(2014)053 [arXiv:1410.4545 [hep-ph]].

[70] G. Burdman, E. Golowich, J. L. Hewett and S. Pakvasa, Phys. Rev. D 66 (2002) 014009 doi:10.1103/PhysRevD.66.014009 [hep-ph/0112235].

[71] E. Golowich, J. Hewett, S. Pakvasa and A. A. Petrov, Phys. Rev. D 79 (2009) 114030 doi:10.1103/PhysRevD.79.114030 [arXiv:0903.2830 [hep-ph]].

[72] C. Greub, T. Hurth, M. Misiak and D. Wyler, Phys. Lett. B 382 (1996) 415 doi:10.1016/0370-2693(96)00694-6 [hep-ph/9603417]. 
[73] S. Fajfer, P. Singer and J. Zupan, Phys. Rev. D 64 (2001) 074008 doi:10.1103/PhysRevD.64.074008 [hep-ph/0104236].

[74] A. Paul, I. I. Bigi and S. Recksiegel, Phys. Rev. D 82 (2010) 094006 Erratum: [Phys. Rev. D 83 (2011) 019901] doi:10.1103/PhysRevD.83.019901, 10.1103/PhysRevD.82.094006 [arXiv:1008.3141 [hep-ph]].

[75] N. K. Nisar et al. [Belle Collaboration], Phys. Rev. D 93 (2016) no.5, 051102 doi:10.1103/PhysRevD.93.051102 [arXiv:1512.02992 [hep-ex]].

[76] S. Fajfer, N. Kosnik and S. Prelovsek, Phys. Rev. D 76 (2007) 074010 doi:10.1103/PhysRevD.76.074010 [arXiv:0706.1133 [hep-ph]].

[77] S. de Boer and G. Hiller, Phys. Rev. D 93 (2016) no.7, 074001 doi:10.1103/PhysRevD.93.074001 [arXiv:1510.00311 [hep-ph]].

[78] J. M. Zhang and G. L. Wang, Eur. Phys. J. C 71 (2011) 1715 doi:10.1140/epjc/s10052-011-1715-1 [arXiv:1003.5570 [hep-ph]].

[79] H. B. Li and M. Z. Yang, Eur. Phys. J. C 59 (2009) 841 doi:10.1140/epjc/s10052-008-0828-7 [arXiv:0709.0979 [hep-ph]].

[80] A. Khodjamirian, T. Mannel and A. A. Petrov, JHEP 1511 (2015) 142 doi:10.1007/JHEP11(2015)142 [arXiv:1509.07123 [hep-ph]].

[81] A. Badin and A. A. Petrov, Phys. Rev. D 82 (2010) 034005 doi:10.1103/PhysRevD.82.034005 [arXiv:1005.1277 [hep-ph]].

[82] Y.-T. Lai et al. [Belle Collaboration], Phys. Rev. D 95 (2017) no.1, 011102 doi:10.1103/PhysRevD.95.011102 [arXiv:1611.09455 [hep-ex]].

[83] A. J. Buras, D. Buttazzo, J. Girrbach-Noe and R. Knegjens, JHEP 1511 (2015) 033 doi:10.1007/JHEP11(2015)033 [arXiv:1503.02693 [hep-ph]].

[84] A. J. Buras, D. Buttazzo and R. Knegjens, JHEP 1511 (2015) 166 doi:10.1007/JHEP11(2015)166 [arXiv:1507.08672 [hep-ph]].

[85] A. V. Artamonov et al. [BNL-E949 Collaboration], Phys. Rev. D 79 (2009) 092004 doi:10.1103/PhysRevD.79.092004 [arXiv:0903.0030 [hep-ex]].

[86] Y. Grossman and Y. Nir, Phys. Lett. B 398 (1997) 163 doi:10.1016/S0370-2693(97)00210-4 [hep-ph/9701313].

[87] C. Smith, arXiv:1409.6162 [hep-ph].

[88] G. Isidori and R. Unterdorfer, JHEP 0401 (2004) 009 doi:10.1088/1126-6708/2004/01/009 [hep-ph/0311084].

[89] The LHCb Collaboration [LHCb Collaboration], LHCb-CONF-2016-012, CERN-LHCb-CONF-2016-012.

[90] A. C. Nayak and P. Jain, arXiv:1610.01826 [hep-ph].

[91] A. M. Baldini et al. [MEG Collaboration], Eur. Phys. J. C 76 (2016) no.8, 434 doi:10.1140/epjc/s10052-016-4271-x [arXiv:1605.05081 [hep-ex]].

[92] Y. Amhis et al., arXiv:1612.07233 [hep-ex]. 